\title{
Intervención sobre las conductas clínicas para mejorar el cuidado obstétrico relacionado al parto: un estudio con- trolado randomizado en racimos (1)
}

\author{
Althabe F, Buekens P, Bergel E, Belizán JM, Campbell MK, Moss N, Hartwell T, Wright \\ LL; Guidelines Trial Group. A behavioral intervention to improve obstetrical care. N \\ Engl J Med 2008;358(18):1929-40.
}

Análisis crítico: Cristián Herrera R. ${ }^{1 a}$, Jorge Carvajal C., PhD ${ }^{2}$

\section{RESUMEN (1)}

Antecedentes: La implementación de prácticas obstétricas basadas en evidencia se mantiene como un desafío muy importante. Objetivos: Diseminar e implementar estrategias efectivas en la práctica obstétrica. Métodos: Se randomizaron 19 hospitales en Argentina y Uruguay para recibir una intervención multifactorial sobre conductas clínicas, con el fin de desarrollar e implementar guías clínicas para el uso de la episiotomía y el manejo activo de la tercera etapa del parto o para no recibir intervención alguna. La intervención incluyó la selección de opiniones de líderes, seminarios interactivos, manuales de entrenamiento de habilidades, visitas de académicos uno-a-uno con los clínicos que atienden los partos, recordatorios y retro-alimentación. El resultado primario fueron las tasas de uso profiláctico de ocitocina durante la tercera etapa del parto y de episiotomía. El principal resultado secundario fue la hemorragia postparto y la calificación de los clínicos que atienden los partos para cambiar su conducta con respecto a la episiotomía y el manejo de la tercera etapa del parto. Los resultados fueron medidos antes de la intervención, luego de 18 meses de intervención y 12 meses después de terminada la intervención. Resultados: La tasa de uso profiláctico de ocitocina en los hospitales que recibieron intervención aumentó de 2,1\% antes de la intervención a 83,6\% luego del término de ella, y de $2,6 \%$ a $12,3 \%$ en los hospitales control $(p=0,01$ para la diferencia en los cambios). La tasa de de uso de episiotomía disminuyó de $41,1 \%$ a $29,9 \%$ en los hospitales que recibieron la intervención, pero se mantuvieron estables en los hospitales control, con valores pre-intervención y post-intervención de $43,5 \%$ y $44,5 \%$, respectivamente $(p<0,01$ para la diferencia en los cambios). La intervención también se asoció a reducción en la tasa de hemorragia postparto de $500 \mathrm{ml}$ o más (tasa relativa de reducción, 70\%; 95\% IC 16 a 78). La calificación de los clínicos que atienden los partos para cambiar su conducta también aumentó en los hospitales que recibieron la intervención. Los efectos en el uso de episiotomía y ocitocina profiláctica se mantuvieron a los 12 meses de terminada la intervención. Conclusión: Una intervención conductual multifactorial aumentó el uso profiláctico de ocitocina durante la tercera etapa del parto y redujo el uso de episiotomía.

\section{ANÁLISIS DE LA INVESTIGACIÓN}

\section{A. Relevancia clínica de la investigación}

Para mejorar el cuidado obstétrico relacionado al parto, ¿es útil una intervención conductual multifactorial en un centro hospitalario? Para los resultados utilizados como parámetros en este estudio, 
sabemos que la episiotomía profiláctica de rutina tiene mayores riesgos de: trauma perineal posterior, uso de suturas y complicaciones en la cicatrización, que los protocolos de uso restrictivo de episiotomía (2). En cuanto al uso de ocitocina profiláctica en la tercera etapa del embarazo, sabemos que previene la hemorragia postparto y que disminuye el uso de oxitócicos terapéuticos, constituyendo un hecho beneficioso para el parto (3). Ambas conductas clínicas son de uso variable, y es deseable incrementar su práctica.

\section{B. El estudio (1)}

Diseño: Estudio controlado randomizado en racimos, con los hospitales como unidad de randomización. Fueron incluidas 19 maternidades en hospitales públicos de Argentina y Uruguay que tuvieran al menos 1000 partos vaginales al año, sin protocolos para episiotomías selectivas ni manejo activo de la tercera etapa del parto, y tasas de episiotomías sobre $20 \%$ y de uso profiláctico de ocitocina menor a $25 \%$ en partos de mujeres con fetos únicos. La randomización se realizó con un "procedimiento balanceado de randomización".

Intervención: La intervención duró 18 meses. Equipos de 3 a 6 clínicos que atendían los partos (médicos, residentes o matronas) fueron entrenados en un seminario de 5 días para desarrollar y diseminar guías clínicas basadas en evidencia, sobre el manejo de la tercera etapa del parto y el uso de la episiotomía. Al regresar a sus respectivos hospitales, los equipos participaron en un seminario de 1 día para desarrollar sus habilidades de entrenamiento. Luego, los equipos diseminaron las guías, entrenaron y visitaron a los clínicos que atienden los partos, y crearon recordatorios que se pusieron en las salas de parto, dentro de paquetes quirúrgicos para los que clínicos que atienden los partos, y en las fichas clínicas.

Medición de resultados: Los resultados primarios fueron las tasas de uso de ocitocina profiláctica (dosis única de $10 \mathrm{UI}$ ev durante la tercera etapa del parto) y las tasas de episiotomía en partos vaginales únicos. Los resultados fueron medidos en condición basal ( 3 meses antes de la randomización), al término de la intervención (3 últimos meses de los 18 meses de intervención), y al año de seguimiento ( 3 meses luego de 1 año). Los resultados secundarios fueron la tasa de uso de sutura perineal, tasas de hemorragia postparto de $500 \mathrm{ml}$ o más y $1000 \mathrm{ml}$ o más, y la calificación de los clínicos que atienden los partos para cambiar su conducta con respecto a la episiotomía y el manejo de la tercera etapa del parto.
Resultados: El uso profiláctico de ocitocina en los hospitales que recibieron intervención aumentó significativamente. La tasa de uso de episiotomía disminuyó en los hospitales que recibieron la intervención pero se mantuvieron estables en los hospitales control. El porcentaje de partos con hemorragia postparto significativa $(\geq 500 \mathrm{ml}$ ) se redujo con la aplicación de la intervención (Tabla I). Durante la evaluación en el seguimiento luego de 1 año, se registró una tasa de uso profiláctico de ocitocina de $73,3 \%$ y $7,1 \%$, respectivamente, con diferencia absoluta en la tasa cambio en comparación con la línea de base de 63,9\% (95\% IC, -3,8 a 80; $p=0,08$ ). El uso de episiotomía en los hospitales con intervención y en los hospitales control, fue $28,1 \%$ y $45,1 \%$ respectivamente, con una diferencia absoluta en la tasa de cambio de $-8,7 \%$ (95\% IC, $-18,1$ a $-4,3 ; p=0,004)$. La calificación para cambiar la conducta clínica fue medida después del término de la intervención en 617 clínicos que atendían los partos (324 y 293 en cada grupo), mostrando un aumento de $14,4 \%$ a $55,9 \%$ en los hospitales con intervención, manteniéndose estable en los hospitales control, registrándose una tasa de diferencia en lo cambios de $38,4 \%$ (95\% IC, 19,6 a 56,9 ; $p<0,001)$. No se registraron diferencias significativas en otros resultados como extracción manual de la placenta, suturas perineales (solo una disminución en los desgarros de $2^{\circ}$ grado), mortinatos, puntaje de Apgar a los 5 minutos, mortalidad neonatal, admisión materna a $\mathrm{UCl}$ o mortalidad materna.

\section{Análisis crítico}

Validez interna: El estudio es randomizado, y el análisis se efectuó según intención de tratar; no explicita si tuvo ocultamiento de la secuencia de randomización, y se utilizó un "procedimiento balanceado de randomización" en el cual la asignación de los hospitales a la intervención o a control fueran balanceados con respecto a las tasas de uso profiláctico de ocitocina y episiotomía, la presencia o ausencia de programas de residencia, el país y la región donde estaba ubicado el hospital, y el número anual de nacimientos en el hospital. El estudio consiguió un buen seguimiento, variando por resultados, desde $99,5 \%$ de seguimiento en los nacimientos a $96,3 \%$ de seguimiento en los datos de hemorragia postparto. El uso de ciegos no era posible en los clínicos, pero donde si podía ser usado (pacientes, recolectores, adjudicadores, analistas), no fue especificada su aplicación. El análisis de datos fue apropiado, especialmente porque se utilizaron los hospitales como unidad de análisis.

Comentarios: En términos generales el estudio 
Tabla I

\section{EFECTOS DE LA INTERVENCIÓN SOBRE CONDUCTAS CLÍNICAS EN LAS TASAS DE USO PROFILÁCTICO DE OCITOCINA DURANTE LA TERCERA ETAPA DEL PARTO, EN LAS TASAS DE EPISIOTOMÍA Y EN EL PORCENTAJE DE HEMORRAGIA POSTPARTO}

\begin{tabular}{|c|c|c|c|c|c|c|c|c|}
\hline \multirow[t]{2}{*}{ Outcome } & \multicolumn{3}{|c|}{$\begin{array}{l}\text { Hospitales con intervención } \\
\qquad(\mathrm{n}=10)\end{array}$} & \multicolumn{3}{|c|}{$\begin{array}{l}\text { Hospitales control } \\
\qquad(\mathrm{n}=9)\end{array}$} & \multicolumn{2}{|c|}{$\begin{array}{l}\text { Efecto de la } \\
\text { intervención }\end{array}$} \\
\hline & $\begin{array}{c}\text { Tasa } \\
\text { línea de } \\
\text { base } \\
(n=2963)\end{array}$ & $\begin{array}{c}\text { Tasa post - } \\
\text { intervención } \\
(\mathrm{n}=2587)\end{array}$ & $\begin{array}{l}\text { Tasa } \\
\text { de } \\
\text { cambio }\end{array}$ & $\begin{array}{c}\text { Tasa } \\
\text { línea de } \\
\text { base }^{\star} \\
(n=2503)\end{array}$ & $\begin{array}{l}\text { Tasa post - } \\
\text { intervención } \\
(\mathrm{n}=2366)\end{array}$ & $\begin{array}{c}\text { Tasa } \\
\text { de } \\
\text { cambio* }\end{array}$ & $\begin{array}{l}\text { Diferencia } \\
\text { absoluta en } \\
\text { la tasa de } \\
\text { cambio** }^{\star *}(95 \% \mathrm{IC})\end{array}$ & Valor $p$ \\
\hline $\begin{array}{l}\text { Oxitocina } \\
\text { profiláctica }\end{array}$ & 2,1 & 83,6 & 77,2 & 2,6 & 12,3 & 9,8 & $\begin{array}{c}67,5(38,9 a \\
87,1)\end{array}$ & 0,01 \\
\hline Episiotomía & 41,1 & 29,9 & $-12,7$ & 43,5 & 44,5 & $-1,9$ & $\begin{array}{c}-10,9(-16,1 \\
a-5,8)\end{array}$ & $<0,001$ \\
\hline $\begin{array}{l}\text { Episiotomía } \\
\text { en } \\
\text { primigestas }\end{array}$ & 84,8 & 66,5 & $-21,2$ & 84,1 & 84,6 & $-0,8$ & $\begin{array}{c}-20,4 \quad(-28,9 \\
a-3,3)\end{array}$ & 0,02 \\
\hline $\begin{array}{l}\text { Episiotomía } \\
\text { en } \\
\text { multíparas }\end{array}$ & 18,4 & 12,4 & $-6,8$ & 16,2 & 19,3 & $-1,2$ & $\begin{array}{c}-5,7(-15,1 \mathrm{a} \\
-0,8)\end{array}$ & 0,01 \\
\hline $\begin{array}{l}\text { Hemorragia } \\
\text { postparto } \\
\geq 500 \mathrm{ml} \\
\text { (\%) }\end{array}$ & 18,6 & 6,9 & 0,31 & 9,8 & 8,1 & 0,55 & $\begin{array}{c}0,55(0,29 a \\
0,91)\end{array}$ & 0,03 \\
\hline $\begin{array}{l}\text { Hemorragia } \\
\text { postparto } \\
\geq 1000 \mathrm{ml} \\
(\%)\end{array}$ & 3,0 & 0,8 & 0,26 & 1,5 & 0,6 & 0,88 & $\begin{array}{c}0,3(0,22 \mathrm{a} \\
0,84)\end{array}$ & 0,007 \\
\hline
\end{tabular}

* La tasa de cambio es el promedio de las diferencias entre la tasa post-intervención y la tasa de la línea de base para cada hospital. **La diferencia absoluta en la tasa de cambio es la diferencia entre la tasa de cambio en los hospitales con intervención y la tasa de cambio en los hospitales control.

evitó el sesgo, mediante medidas como: la randomización con procedimiento balanceado, el buen seguimiento, el análisis según intención de tratar y el usar los hospitales como unidad de análisis (con lo que se evita el "error por unidad de análisis", error muy frecuente en estudios randomizados en racimo). Sin embargo, existen factores como: falta de ocultamiento de la secuencia de randomización (potencialmente) y la ausencia de ciegos, que constituyen fuentes de sesgo. Esto último, puede ser importante ya que la asignación de resultados en cada hospital pudo haber sido muy diversa y tendenciosa al no existir ciegos; se explicita, sin embargo, que hubo personal entrenado para evitar este problema. Desde el punto de vista metodológi$c o$, podemos afirmar que el estudio tiene un riesgo moderado de sesgo.

Conclusión: Este estudio demuestra que una intervención multifactorial intensiva puede modificar en el corto (durante la aplicación de la intervención) y mediano plazo (1 año después) las conductas clínicas en el ámbito de la obstetricia en relación al parto. Consideramos que este resultado es válido para el ámbito chileno, ya que el escenario del estudio fueron países latinoamericanos en vías de desarrollo, aunque la estructura de los sistemas de salud es distinta al nuestro. En cuanto a la aplicabilidad del estudio, reconocemos la complejidad de la intervención, pero el resultado apoya la evidencia que demuestra que estas son las intervenciones efectivas para cambiar la conducta de los clínicos $(4,5)$. Se requiere evaluar el costo-efectividad de 
estas intervenciones y su reproducibilidad a otras áreas geográficas, culturales, políticas y otras áreas de práctica clínica. Igualmente se requiere estudiar si intervenciones menos complejas pueden obtener el mismo beneficio, a menor costo.

\section{BIBLIOGRAFÍA}

1. Althabe F, Buekens $P$, Bergel E, Belizán JM, Cambell MK, Moss N, Hartwell T,Wright LL; Guidelines Trial Group. A behavioral intervention to improve obstetrical care. N Engl J Med 2008;358(18):1929-40.
2. Carroli G, Belizán J. Episiotomy for vaginal birth. Cochrane Database of Systematic Review. 2000;(2): CD000081.

3. Cotter A, Ness A, Tolosa J. Prophylactic oxytocin for the third stage of labour. Cochrane Database Syst Rev. 2001;(4):CD001808.

4. Gülmezoglu AM, Langer A, Piaggio G, Lumbiganon $P$, Villar J, Grimshaw J. Cluster randomized trial of an active, multifaceted educational intervention based on the WHO Reproductive Health Library to improve obstetric practices. BJOG 2007;114:16-23.

5. Thorp J. O', Evidence-based medicine-where is your effectiveness? BJOG 2007;114(1):1-2. 